Revue internationale P.M.E.

Économie et gestion de la petite et moyenne entreprise

\title{
Autorité et pratiques d'animation des relations humaines des dirigeants de TPE dans un éthos méditerranéen
}

\section{Soufyane Frimousse}

Volume 25, numéro 1, 2012

URI : https://id.erudit.org/iderudit/1014872ar

DOI : https://doi.org/10.7202/1014872ar

Aller au sommaire du numéro

Éditeur(s)

Presses de l’Université du Québec

ISSN

0776-5436 (imprimé)

1918-9699 (numérique)

Découvrir la revue

Citer cet article

Frimousse, S. (2012). Autorité et pratiques d'animation des relations humaines des dirigeants de TPE dans un éthos méditerranéen. Revue internationale P.M.E., 25(1), 13-38. https://doi.org/10.7202/1014872ar
Résumé de l'article

La région nord du Maroc évolue avec les effets de la " mondialisation », la montée concomitante de l'individualisme... Toutefois, la dimension communautaire constitue encore le noyau dur de cette région. Les valeurs ne se délitent pas, mais évoluent et se transforment. Cette dimension communautaire et les valeurs qui les accompagnent se transposent-elles dans les pratiques des dirigeants de toutes petites entreprises ? Comment le dirigeant parvient-il à concilier, dans son style d'encadrement et ses pratiques d'animation des relations humaines, le halo de valeurs puisé dans les permanences du passé et les évolutions sociétales ? Comment s'opère la complexe articulation entre l'universel et le contextuel ? Notre contribution s'attache à expliquer comment le fonds culturel est préservé par le dirigeant et pourquoi il a encore un effet social et une signification. 


\title{
Autorité et pratiques d'animation des relations humaines des dirigeants de TPE dans un éthos méditerranéen
}

Soufyane FRIMOUSSE IAE de Corse

MOTS CLÉS

Autorité - Dirigeant - TPE - Pratique d'animation des relations humaines - Éthos méditerranéen

\begin{abstract}
L'AUTEUR
SOUFYANE FRIMOUSSE est maître de conférences et détient un doctorat en sciences de gestion. Ses domaines d'expertise sont le comportement organisationnel, la gestion du changement, les modèles de management, le climat social, le management de la diversité, l'audit social et de RSE. Membre de l'AGRH, de l'IAS et de l'ANDRH. Il a contribué à plusieurs ouvrages collectifs et à de nombreuses revues scientifiques et professionnelles, françaises et internationales. Adresse: IAE de Corse, 7, avenue Jean-Nicoli, 20250 Corte, Corse. Courriel: $<$ frimousse@univ-corse.fr>.
\end{abstract}

\section{RÉSUMÉ}

La région nord du Maroc évolue avec les effets de la «mondialisation», la montée concomitante de l'individualisme... Toutefois, la dimension communautaire constitue encore le noyau dur de cette région. Les valeurs ne se délitent pas, mais évoluent et se transforment. Cette dimension communautaire et les valeurs qui les accompagnent se transposent-elles dans les pratiques des dirigeants de toutes petites entreprises? Comment le dirigeant parvient-il à concilier, dans son style d'encadrement et ses pratiques d'animation des relations humaines, le halo de valeurs puisé dans les permanences du passé et les évolutions sociétales? Comment s'opère la complexe articulation entre l'universel et le contextuel? Notre contribution s'attache à expliquer comment le fonds culturel est préservé par le dirigeant et pourquoi il a encore un effet social et une signification. 


\begin{abstract}
The Northern part of Morocco is changing due to globalization and the increasing individualism that comes with it. However, that region still has a strong sense of community. Values are not disappearing, they are just changing. Can this sense of community and these values be also found in very small companies? How do managers combine, in the way they run their business and they care for their employees, the circle of values kept from the past and those taken emerging from our modern society? How does the complex relationship between what is universal and what is contextual work? We will try to explain how company managers keep their cultural background and why it is still influential and meaningful.
\end{abstract}

\title{
RESUMEN
}

El norte de Marruecos está cambiando con los efectos de la "globalización ", lo cual ha provocado un aumento del individualismo... Sin embargo, el comunitarismo sigue caracterizando esta región. Los valores no se han desintegrado, pero se desarrollan y cambian. ¿Cómo se incorporan la dimensión comunitaria y los valores que la acompañan en las prácticas de los líderes de pequeñas empresas? ¿Cómo concilian los líderes en su estilo de liderazgo las prácticas de manejo de relaciones humanas, los valores provenientes de tiempos pasados y la evolución social? ¿Cómo tiene lugar esta compleja articulación entre lo universal y lo contextual? Nuestra contribución tiene como objetivo explicar cómo lo cultural sigue siendo custodiado por líderes empresariales y por qué esto tiene un efecto social con profundo sentido.

\section{Introduction}

Dans les toutes petites entreprises (TPE), le dirigeant s'appuie généralement sur un système d'autorité et d'encadrement, car il pense qu'il doit accompagner son pouvoir et ses responsabilités. Weber (1956) définit le pouvoir comme la capacité pour un individu de faire prévaloir sa volonté. L'exercice du pouvoir réclame l'autorité qui correspond à la capacité à faire exécuter un commandement. L'autorité n'est stabilisée que si elle est légitime. Weber (1956) distingue trois formes d'autorité : celle qui émane des personnalités charismatiques, celle qui se fonde dans la tradition et l'autorité rationnelle. Le cadre wébérien permet d'appréhender les fondements de la légitimité des dirigeants. Toutefois, il ne peut nous économiser la réflexion sur d'autres modes interprétatifs. Il s'agit notamment d'aborder la question de l'autorité et de l'encadrement en évaluant la légitimité des dirigeants à partir de la dimension contextuelle. 
En Méditerranée du Sud et en Afrique du Nord, le monde domestique familial reste souvent le cadre dominant; famille et traditions-coutumes ${ }^{1}$ sont encore des référents prégnants. Les positions hiérarchiques et les chaînes de dépendance sont très importantes. L'individu ne peut être dissocié de son appartenance à un corps social plus large, à un groupe; il est un maillon dans une chaîne d'êtres. L'identification et la fidélité à un groupe sont essentielles. La personne est «identitairement» liée à une famille et à un milieu social. Le père est l'incarnation de la grandeur et de la tradition; ses appréciations, ses distinctions et ses considérations renvoient aux jugements d'un supérieur ou d'un chef. Il est censé se montrer responsable et bienveillant, en toutes circonstances, à l'égard de son entourage. Certes, les sociétés méditerranéennes évoluent avec les effets de la "mondialisation», la montée concomitante de l'individualisme et la prépondérance des considérations financières... Toutefois, la dimension communautaire constitue encore le noyau dur de la société des régions qui nous concernent. Les valeurs ne se délitent pas, mais évoluent et se transforment.

Cette dimension communautaire et les valeurs qui les accompagnent se transposent-elles dans les pratiques des dirigeants des TPE, où le facteur petite taille et facilité de contacts directs jouent un rôle non négligeable? Comment le dirigeant peut-il concilier, dans ses pratiques d'animation des relations humaines ${ }^{2}$, le halo de valeurs puisé dans les permanences du passé et les évolutions sociétales? Comment s'opère la complexe articulation entre l'universel et le contextuel? Comment le legs d'une époque globalement révolue agit-il encore sur les logiques d'action et d'exécution du dirigeant dans un contexte en mutation?

Notre contribution s'efforce d'expliquer comment le fonds culturel continue à être conservé par le dirigeant et pourquoi il a encore un effet social et fait sens. Sur quoi se fonde la légitimité du dirigeant et de ses actions? Comment (fonctionnalité) et pourquoi (le sens/la finalité) dirige-t-il son équipe autrement que ce à quoi nous a habitués le management issu de la littérature managériale?

Afin de répondre à ces interrogations, nous commençons par une clarification des concepts étudiés: le dirigeant, la TPE, le mix social. Il s'agit surtout

1. La tradition renvoie à l'idée d'un héritage culturel. Il s'agit de la représentation d'un contenu significatif conservé dans le temps, transmis par un mode particulier, doté d'une force agissante et d'une prédisposition à la reproduction (Lenclud, 2007).

2. Les services des ressources humaines ne sont pas très développés dans les TPE; néanmoins, le dirigeant assure une fonction d'animation et de régulation des relations humaines. 
de présenter les grandes caractéristiques du dirigeant au sein des TPE ainsi que le mix social proposé par Mahé de Boislandelle (1998). Cette approche est revisitée et adaptée aux TPE par Fourcade, Gallego, Polge et Saoudi (2010) qui proposent le concept de mix de relations humaines. Nous avons également choisi comme point d'appui les travaux menés par Marchesnay (2002) pour comprendre les logiques d'action du dirigeant.

Durant les années 1970, deux approches d'analyse des TPE se sont développées: le courant de la spécificité des entreprises de petite taille et celui de la diversité qui cherche à établir des typologies dans le but d'ordonner et de classer l'hétérogénéité du monde des TPE. Notre recherche s'insère dans le courant de la spécificité. La prise en compte de la dimension contextuelle dans une théorie de l'entrepreneuriat comprend le rôle des valeurs dominantes d'une communauté (l'éthos), l'histoire, la géographie des territoires. Il s'agit d'approfondir la dimension territoire/milieu telle que préconisée par Julien, Marchesnay et Madado (2010); Brinckmann, Grichnik et Kapsa (2010); Serarols, Aldemir et Joo (2011). Ces chercheurs soutiennent que le dirigeant est immergé dans un contexte unique dans lequel interfèrent des considérations qui agissent sur ses actions et ses pratiques.

Dans le cadre de nos recherches, nous considérons que le fonds culturel de la région nord du Maroc Tanger/Tétouan s'insère dans un éthos commun méditerranéen qui correspond à un ensemble de valeurs et d'attitudes. Nous reconnaissons un halo de valeurs communes qui rendent les sociétés méditerranéennes comparables: la répartition des rôles entre l'homme et la femme, l'honneur, la honte, la pudeur, la violence et les pratiques vindicatives, un univers social et affectif basé sur le contact avec autrui, le sens de la mesure, le culte des «saints», "vierges» et «des martyrs» (Blok, Bromberger et Albera, 2001). Il s'agit de saisir comment s'opère la liaison entre sphères privées (marquée par l'individualisme et le besoin d'autonomie), l'éthos méditerranéen (le substrat de valeurs communes) et l'action du dirigeant auprès de son personnel.

Par la suite, le cadre méthodologique de la recherche est présenté. Il semble approprié de parler de démarche ethnosociologique ou ethnographique puisque l'objectif est de saisir la logique d'action du dirigeant (vision, intention, buts) et sa quête de légitimité (fonction sociale remplie par l'entreprise et son dirigeant) à travers l'étude de ses pratiques d'animation des relations humaines (emploi, rémunération, valorisation et participation).

Enfin, les premiers résultats sont présentés et discutés. Le dirigeant des TPE est généralement considéré par la doxa managériale comme étant 
paternaliste et/ou «omnipotent». Nous tenterons ici d'aller au-delà de ces constats et de mettre au jour, si possible, d'autres traits distinctifs insistant plus sur des notions telles que la «fluidité relationnelle» et l' «échange social».

\section{Le dirigeant au sein de la TPE}

La très petite entreprise (TPE) n'a que quelques employés. Elle se caractérise par une stratégie intuitive ou peu formalisée, un système d'information interne et externe très simple (Julien, 1997; Fourcade et al., 2010). Plus la taille de l'entreprise est petite, plus la centralisation du pouvoir opérée par le dirigeant sera forte. Mahé de Boislandelle (1998) qualifie ce phénomène d'effet de grossissement. Le rôle du dirigeant est prédominant. Il impulse l'action et l'exécution notamment dans le cadre des pratiques managériales (Paradas, 2007). Il est nommé chef d'entreprise, patron, entrepreneur, par opposition au cadre ou à l'ingénieur-directeur. Il évolue dans un bouquet de relations de proximité englobant les fonctions et la hiérarchie au sein de l'entreprise, le territoire, la société (Marchesnay, 2003, 2008). La diversité des TPE reflète des cultures et des réalités très différentes d'un secteur à l'autre : agriculture, agroalimentaire, secteurs manufacturiers ou technologiques, commerces de détail... Cette variété nécessite donc des expertises dissemblables. Toutefois, il est possible de distinguer des caractéristiques communes.

\section{1. Égotrophie, proximité et mix de relations humaines}

Pour Torrès et Gueguen (2008), chacune des caractéristiques managériales associées aux TPE repose sur le principe de proximité. Dans les TPE, le dirigeant-propriétaire est omniprésent dans tous les domaines d'activité. Torrès (2008) nomme ce phénomène l'effet d'égotrophie. La faible taille de ces entreprises favorise les contacts directs et personnels dans l'animation des relations humaines. Il s'agit d'une forme de proximité hiérarchique. Au sein des TPE, toutes les fonctions sont intégrées et fortement reliées. La proximité entre les acteurs permet la polyvalence des salariés. Le «savoir tout faire» est la base du travail. Les multiples contacts aident à la connaissance des divers problèmes de l'entreprise et la flexibilité permet une adaptation rapide aux variations de l'environnement. Cette caractéristique correspond à la proximité intrafonctionnelle.

Ces proximités influencent les axes du mix social. Cette approche centrée sur les PME retient un modèle de gestion des ressources humaines axé sur l'emploi, la rémunération, la valorisation et la participation. L'emploi renvoie essentiellement au recrutement. Il constitue un domaine réservé du dirigeant qui privilégie le relationnel ou le bouche à oreille. Le départ, 
la création de poste et l'augmentation de l'activité sont les principales sources du processus de recrutement. La capacité de rémunérer et l'ajustement aux pratiques du marché sont les socles des pratiques de rémunération.

Les pratiques de valorisation englobent l'évaluation des salariés, le développement de la formation du personnel et l'amélioration des conditions de travail. Les pratiques de participation reposent sur les systèmes d'information et de communication. Mahé de Boislandelle (1998) établit une relation entre le mix social et les performances sociales et économiques. Ce mix social est d'ailleurs couramment mobilisé dans l'étude de la GRH au sein des PME. Dans le cadre des TPE, Fourcade et Polge (2008) abordent le mix social sous l'angle de l'animation des relations humaines.

La faiblesse de l'effectif renforce la personnalisation des relations dans et hors de l'entreprise. Le recrutement dans les TPE est l'affaire exclusive du dirigeant. Il choisit prioritairement son personnel dans sa famille ou dans ses relations. La rémunération dépend de la capacité et de la santé financière de l'entreprise. La valorisation est informelle, non structurée et permanente. Au regard de la participation, les dirigeants privilégient les pratiques fondées sur la confiance, le contact quotidien, la reconnaissance, les rencontres informelles, etc. (Dejoux, 2001). Les relations interpersonnelles, les liens émotionnels et affectifs qui caractérisent toutes les organisations demeurent donc potentiellement plus intenses et exacerbés dans les TPE. $\mathrm{Au}$ sein de ce type d'organisation, les aspirations des dirigeants transposées dans ses pratiques d'animation des relations humaines ne se limitent pas uniquement à la recherche de croissance ou de création de valeur, certains dirigeants de TPE privilégiant d'autres logiques.

\subsection{Les aspirations, les logiques d'actions du dirigeant et ses pratiques d'animation des relations humaines}

À partir d'une base d'aspirations, Marchesnay (2002) propose plusieurs logiques d'action. La «logique patrimoniale » privilégie la transmission de l'entreprise considérée comme un patrimoine. Afin d'assurer la pérennité $(\mathrm{P})$, le patrimoine doit demeurer dans les sphères familiales, ce qui implique l'indépendance du capital (I), c'est-à-dire le refus de capitaux étrangers. Ces deux dimensions sont les conditions à la poursuite de croissance (C). Ce type de dirigeant est qualifié de PIC. La croissance s'effectue dans des activités stables, fournissant un profit régulier. Le PIC gère les hommes en s'appuyant sur l'affectif. Il est également possible de distinguer des dirigeants de TPE suivant «la logique entrepreneuriale » qui repose sur les activités innovantes à forte croissance (C) potentielle et l'autonomie (A) de décision de l'entrepreneur. La pérennité $(\mathrm{P})$ doit permettre au dirigeant 
de réaliser ses aspirations; dans le cas contraire, elle n'est pas recherchée. Ce profil est désigné CAP. Le CAP recherche la flexibilité maximale. Sa gestion des hommes s'appuie sur la quête d'efficacité et de performance. Il incite et oriente afin d'améliorer les compétences. Il assume les risques stratégiques. À côté de cette distinction duale existe «la logique managériale» qui privilégie les qualités de gestionnaire de l'entrepreneur. Les relations humaines y sont plus anonymes et procédurales. Les dirigeants tendent vers une organisation rationnelle et formalisée. Ils cherchent la maximisation de la valeur de l'entreprise. Le dirigeant-cadre considère son entreprise comme un outil de travail. Les personnes sont appréhendées comme des ressources humaines.

Ces aspirations et ces logiques d'action sont influencées par la dimension contextuelle. D'ailleurs, de récents travaux s'intéressent à l'influence des facteurs culturels sur le dirigeant (Thornton, Ribeiro-Soriano et Urbano, 2011). Les liens entre encastrement territorial et détection des opportunités d'affaires par le dirigeant sont notamment examinés (Korsgaard et Anderson, 2011). Qu'en est-il de l'éthos méditerranéen de la région nord du Maroc? Accentue-t-il les caractéristiques de la TPE? Agit-il sur les pratiques d'animation des relations humaines du dirigeant?

Avant de répondre à ces interrogations, il convient de préciser ce que recouvre la notion d'éthos méditerranéen.

\subsection{Des TPE dans un éthos méditerranéen: un dirigeant "encastré»}

Toutes les caractéristiques de la TPE se rencontrent, à des degrés divers, dans toutes les TPE tangéroises, mais l'on observe aussi d'autres caractères plus spécifiques. Trois cercles agissent sur l'entrepreneur. Le premier regroupe son profil et son parcours, le deuxième est constitué par l'ensemble des valeurs d'une société donnée et le troisième renvoie aux milieux qui suscitent et favorisent l'acte de création et de reprise. Dans le cadre de cette recherche, le deuxième et le troisième cercle sont privilégiés.

Gellner (2003) a proposé un modèle théorique dans lequel différentes dimensions de la société maghrébine s'articulent et sont utilisées comme grille d'analyse: groupes ethniques, Islam rural, Islam urbain... Les travaux de Gellner datent des années 1950 et 1960. Ils s'inscrivaient dans un contexte historique particulier (décolonisation). Ils ont le mérite d'interroger et de s'intéresser à des dimensions bien souvent ignorées. Bien qu'intéressantes, ces analyses semblent en décalage avec les réalités actuelles du Maghreb. En effet, l'individu n'est pas prisonnier d'un groupe qui lui dicte sa conduite. 
Il est simplement relié à différents groupes d'appartenance par la nisba (origine) et la nasab (filiation). Ce sont des processus d'identification sociale participant à l'expression de sa volonté et de sa personnalité.

De nombreux chercheurs ont conservé une image de la région nordmarocaine comme région autosuffisante, locale et homogène dans son organisation sociale, son système de production et de reproduction et dans les pratiques et les conduites qu'elle apprend à ses membres. Ce façonnage de la société dite traditionnelle est tel que chaque personne est considérée comme un être pour autrui et par autrui. L'accent est mis sur la conformité totale que la société exige de ses membres. Il s'agit d'une forme de déterminisme. Or, la société nord-marocaine n'est pas figée ni monolithique; ses membres prennent parfois de la distance avec les normes sociales, qui sont sources de conflits, de contradictions. En tant que chercheur, nous souhaitons être à l'écoute de ces contradictions et débats. Il s'agit de saisir les réponses apportées par la société nord-marocaine aux défis que pose l'ouverture à l'international. Dans cette optique, nous avons choisi l'étude du style d'autorité, d'encadrement et d'animation des relations humaines du dirigeant de TPE. Nous considérons que l'hétérogénéité culturelle de la société nord-marocaine s'insère dans un éthos qui oriente les actions, mais qui ne les détermine pas!

Cet éthos n'interdit pas l'improvisation; il n'impose pas à autrui. Il accepte des singularités, mais dans le cadre d'un sens commun. L'éthos donne la direction et subit les tensions actuelles apportées par des situations nouvelles. Il s'agit des crispations entre liberté et déterminisme, collectivité et individualité, conformisme et singularité... Nous pouvons utiliser la métaphore du jeu et du joueur de Merleau-Ponty ${ }^{3}$ pour expliquer ces reconfigurations incessantes des champs d'action en fonction des dynamiques en cours. En d'autres termes, notre objectif est de bien comprendre les interpénétrations entre les histoires locales et la grande histoire (Berque, 1955), c'est-à-dire les mouvements universels.

Pour Geertz (1973), la culture est assimilable à une toile d'araignée tissée par plusieurs individus. Il y a de multiples enchevêtrements. Ce constat à lui seul infirme l'hypothèse d'un modèle «dogmatique» diffusant des

3. Le terrain de football est parcouru par des lignes de force (les lignes de touche) qui appellent un certain mode d'action, la déclenchent et la portent. Le joueur fait corps avec le terrain. Le terrain de football a des limites bien convenues. Toutefois, le joueur peut modifier l'aspect du terrain en y insérant de nouvelles lignes de force où l'action, à son tour, s'écoule et se réalise. 
normes culturelles à des groupes sociaux supposés passifs. Bourdieu (2001) a contribué à enrichir la connaissance du Maghreb en montrant notamment que les logiques sociales s'enracinent dans la volonté des individus.

Geertz (1973) s'est efforcé d'introduire les systèmes culturels des groupes étudiés pour comprendre le sens que donnent les individus aux symboles et aux signes observables dans la vie quotidienne (Addi, 2004).

Les cultures du Maghreb sont à la fois communes et plurielles. D'un côté se trouvent les Berbères (Kabyles, Chaouia, Rifains, etc.) et de l'autre, les Arabes, mais aussi les métissés berbéris arabes. Pour reprendre l'expression d'Étienne (1999), il s'agit des «grains de grenade» pas tout à fait semblables, mais côte à côte dans le même ensemble. Pour cette raison, nous pensons qu'il est inapproprié de recourir à l'«ethnicité4» comme élément d'analyse. Les Berbères et les Arabes de la région nord du Maroc sont-ils disposés comme les cases d'un échiquier? Cette forme d'organisation binaire a été mise en question par Berque $(1953,1955)$ dans Qu'est-ce qu'une tribu nordafricaine?. Il s'agit plutôt de cercles emboîtés dans une société segmentaire «moi contre mes frères; mes frères et moi contre mon cousin; mes cousins, mes frères et moi contre le monde ». La segmentarité consiste dans le jeu de la fission et de la fusion des éléments du système (Favret-Saada, 1966). Les mutations en cours rendent difficile une analyse en termes d'ethnicité. En outre, l'urbanisation, la scolarisation, les effets de la modernité, la religion engendrent un phénomène complexe: la «détribalisation » des communautés, accompagnée néanmoins de la persistance de l'esprit tribal dans les pratiques sociales. Au-delà des spécificités ethniques et pratiques typiques, ce sont les structures de l'environnement sociétal qui sont identiques: un esprit de corps «açaba $»^{5}$.

De multiples et intenses échanges ont créé un fonds culturel commun qui s'insère dans l'éthos méditerranéen (Frimousse et Peretti, 2006). Bien qu'elle soit une mer fermée, la Méditerranée fonde une appartenance ouverte. Elle est composée de populations ni trop proches, ni trop lointaines. Ainsi, une toile de connivences culturelles permet aux hommes de se connaître et de se reconnaître. En Méditerranée, les relations entre les peuples ont été faites d'affrontements et d'apports réciproques. De ces interactions a résulté un éthos commun qui correspond à un ensemble de valeurs et d'attitudes (la convivialité, le contact avec autrui, le «prendre son temps», le sens de

4. Cf. Allali (2010) et Light et Gold (2000).

5. Pour Ibn Khaldun, l'esprit de corps ne se manifeste que chez les gens qui tiennent ensemble par les liens du sang ou par quelque chose d'analogue. Sous l'influence de ces sentiments, ils se soutiennent les uns les autres, ils se prêtent un mutuel secours ( $c f$. Lacoste, 1998). 
la mesure, l'accord entre l'homme et la nature...). Le travail vise d'abord la satisfaction des besoins élémentaires d'un groupe puis la maximisation des profits personnels. Ce processus favorise un sentiment d'appartenance, d'authenticité dans un réseau social bien déterminé.

$\mathrm{La}$ «pression» sociale et les rapports d'interconnaissance favorisent une forme de «rigidité» comportementale issue d'une logique de l'honneur. La cohésion de la communauté, les relations familiales participent à la représentation collective de l'honneur qui s'observe également dans les relations entre les genres et le rapport à l'autorité (Pitt-Rivers, 1997; Gélard, 2003). La «confrontation» avec l'Autre qui existe dans la même sphère est une constante dans cette partie du monde puisque chacun se définit dans un jeu de miroirs avec son voisin dans les domaines économiques, démographiques, etc. En Méditerranée, la proximité ne réunit pas des civilisations complètement étrangères (Frimousse, 2011).

Malgré les évolutions contemporaines, cet éthos est toujours présent comme toile de fond orientant les conduites quotidiennes et les comportements. Les ethnologues ont contribué à définir les valeurs qui caractérisent les relations sociales dans les pays méditerranéens. Pour autant, ces valeurs sont-elles propres aux sociétés méditerranéennes? Certains chercheurs en doutent. Seulement, voilà, nous pensons que ces valeurs connaissent une accentuation singulière dans la plupart des sociétés méditerranéennes. Elles y sont davantage appliquées avec différentes intensités et modulations.

Être social, produit de son milieu, le dirigeant de TPE intègre-t-il les dimensions contextuelles et notamment la culture dans ses logiques d'action et ses pratiques d'animation de relations humaines? Le dirigeant est enraciné et interconnecté dans un contexte avec ses cultures, son histoire, sa géographie. Comment cet éthos façonne-t-il son système d'autorité et d'encadrement?

\section{Le cadre méthodologique: la capture, la découverte et la compréhension}

Notre recherche sur les dirigeants de TPE interroge le mix de relations humaines, le style d'autorité et d'encadrement ainsi que les logiques d'action des dirigeants tels qu'ils sont perçus et vécus par les acteurs. Dans la Grounded Theory (GT) développée par Glaser et Strauss (1967), le chercheur commence par une collecte de données de terrain et effectue une analyse théorisante. Dans cette perspective, l'objet de recherche est défini comme un «territoire à explorer» (Guillemette, 2006). Cette vision est proche de l'ethnométhodologie par son ancrage dans le terrain. Cette dernière est considérée comme une méthode d'incursion dans des niveaux de la réalité qui échappent 
normalement aux observateurs scientifiques qui s'appuient exclusivement sur des déterminations rationnelles et objectives. L'ethnométhodologie s'éloigne néanmoins de la Grounded Theory puisque l'observation et l'analyse des acteurs sont considérées comme suffisantes. L'ethnométhodologie suppose de ne pas interpréter les faits sociaux à la place des acteurs, mais d'aborder la réalité sociale sans projet a priori, en respectant le savoir et le sens commun des acteurs (Garfinkel, 1957). Selon Glaser et Strauss (1967), la compréhension réclame des analyses, un aller-retour sur le terrain et des comparaisons avec les lectures théoriques et expériences antérieures. Dans le cadre de notre recherche, il semble que la pratique du terrain, sous la forme d'une fréquentation des TPE, des salariés et des dirigeants dont les vies sont influencées par de nouvelles situations sociétales culturelles, soit seule capable de nous mettre à l'écoute des configurations actuelles au regard des styles d'encadrement et des pratiques d'animation des relations humaines.

\subsection{Une démarche ethnosociologique}

Pour notre recherche, il semble approprié de parler de démarche ethnosociologique ou d'ethnographie puisque l'objectif est de comprendre et d'expliquer en interprétant au moyen de théories mobilisées en sciences de gestion $^{6}$. Après la Seconde Guerre mondiale, la tendance ethnosociologique s'affirme à travers les recherches de Gouldner (1960) et Merton (1965). Dans le monde francophone, Crozier (1963) ouvre la voie aux premiers travaux sur la dynamique des organisations dans les années 1960; il sera notamment suivi par Pagès (2002). Au cours des années 1980, de nombreux travaux de terrain auront recours à l'ethnographie (Aktouf, 1986; Berry, 1987). Grâce à une fréquentation longue du milieu étudié et à une observation fine des situations, il est possible de rendre compte de cet univers.

Ces enquêtes permettent de saisir et de réfléchir sur la dynamique sociale induite par les décisions et les pratiques mises en place. Elles facilitent également la compréhension du processus d'appropriation par les acteurs des pratiques, des politiques et des concepts de management. Il s'agit de découvrir, derrière le visible et l'immédiat, le niveau sous-jacent de toute réalité en analysant le cadre étudié et en s'efforçant de s'y immerger. La spécificité des études ethnosociologiques repose donc sur l'immersion du chercheur dans un contexte, et l'observation participante est l'une des modalités privilégiées de recueil de données.

6. Cf. Chanlat (2005) et Vernazobres (2009). 


\subsubsection{Le rôle central de l'observation et de l'entretien semi-directif}

Adler et Rochford (1986) proposent une typologie de l'observation participante fondée sur le degré d'implication du chercheur dans la situation observée. Ils distinguent: l'observation participante périphérique dans laquelle le chercheur ne joue pas de rôle clé dans la situation; l'observation participante active impliquant que le chercheur joue un rôle précis et formel dans l'organisation sans être un acteur à part entière; l'observation participante complète correspondant à une situation dans laquelle le chercheur est déjà impliqué en tant qu'acteur dans les situations étudiées. Dans le cadre de nos recherches, nous optons pour l'observation participante périphérique, car elle facilite la mise en place d'une distance suffisante par rapport à l'objet de l'étude nécessaire à l'analyse. Il s'agit de familiarité distante permettant l'acceptation par un groupe. Cette dernière réclame de saisir les dimensions implicites, une bonne ouverture d'esprit, des qualités d'écoute et de mise en retrait (Chanlat, 2005), l'objectif étant de ne pas être perçu comme un cheval de Troie de tel ou tel sous-groupe.

Suivant les recommandations de Spradley (1980), notre étude s'est appuyée sur l'observation des activités, des personnes et des éléments physiques liés à la situation. Le rapport d'étonnement avec éveil de l'attention est fort utile. L'approche par angle ouvert s'inscrit dans la même dynamique. Il s'agit de capter le spectre d'information le plus large possible. Le chercheur observateur tient en permanence un cahier de notes sur lequel il rédige des observations objectives et des sentiments et sensations personnels. Après quelques périodes d'observation, il lui est possible de constituer des repères et des systèmes de décodage, de comprendre quelques idiosyncrasies, de situer les personnes et les relations. L'entretien individuel semi-directif complète ses observations. Une telle étude exploratoire ne peut être conduite à l'aide d'un questionnaire administré par correspondance. L'entretien semidirectif permet d'accéder aux faits, aux représentations et aux interprétations sur des situations connues par les acteurs.

Le dirigeant ne se «livre» qu'à l'issue d'un long processus et de multiples rencontres. Les «bonnes informations» sont celles qui sont tues et implicites. Une telle étude est complexe, car deux grands acteurs y interfèrent avec des enjeux différents (dirigeant/salariés), d'où la nécessité de trianguler les informations et de recourir à l'observation. L'immersion a duré deux semaines dans chaque TPE. Il faut d'abord assister régulièrement aux journées de travail et aux réunions vécues comme routinières ou fastidieuses par les salariés et les dirigeants pour accéder aux conversations informelles qui sont une source incomparable d'informations. Ces étapes peuvent servir d'accélérateur dans la compréhension du décalage existant entre la scène 
et les coulisses. L'accès à un discours moins contrôlé se paie par ailleurs par une difficulté à poser certaines questions dont on sait qu'elles vont mettre mal à l'aise, c'est le cas des questions relatives aux rétributions attendues. L'idéal d'une relation à priori distanciée n'a pas résisté à la pratique. Bien qu'ayant bénéficié à nos débuts d'une forme d'indifférence bienveillante de la part des dirigeants et des salariés, la proximité culturelle nous a permis de tisser des relations mêlant intérêt du chercheur et affect. Toute la difficulté étant de ne pas tomber dans l'objet de la recherche et d'ainsi perdre son objectivité (Bachelot, 2011).

\subsubsection{Les buts, l'organisation et le contexte}

Notre approche méthodologique (entretiens semi-directifs/guide d'entretien ${ }^{7}$ et observations au sein de l'entreprise pendant les activités de production) repose sur la compréhension du style d'autorité et d'encadrement du dirigeant ainsi que sur ses pratiques d'animation de relations humaines. Dans cette optique, les dimensions définies par Marchesnay (2004) sont utilisées comme grille: les buts, le contexte et l'organisation. La dimension «buts» aide à saisir la logique d'action du dirigeant; elle est essentielle à la compréhension (pourquoi ?). La dimension «organisation» s'intéresse aux pratiques d'animation des relations humaines. Quelles sont les pratiques? Sont-elles efficaces (l'atteinte des objectifs), efficientes (minimisation des coûts) et effectives (satisfaction de l'ensemble des membres de l'organisation)? La dimension «contexte» est importante pour apprécier le degré de légitimité. Cette dernière correspond à l'adhésion ou conformité aux valeurs sociétales. L'autorité et les pratiques d'animation et de gestion des relations humaines du dirigeant sont-elles acceptées? Sur quels éléments repose cette légitimité?

\subsection{Présentation de l'échantillon et traitements des données}

Notre échantillon est formé de 15 dirigeants de TPE de la région nord du Maroc (Tanger/Tétouan) et de 15 salariés évoluant dans ces entreprises. Les TPE présentent l'avantage de permettre d'identifier rapidement les lieux clés de l'observation, les «gardiens» $\left(\right.$ gatekeepers $\left.^{8}\right)$ incontournables. Il est plus aisé

7. Dans cette recherche, le guide d'entretien se compose de questions ouvertes. Les entretiens d'une durée d'environ une heure sont réalisés à la date et aux heures retenues par les personnes interrogées au sein de l'entreprise ou à l'extérieur. Avec l'accord des interviewés, les entretiens sont enregistrés sur magnétophone afin de conserver l'ensemble du contenu. Les entretiens suivent quatre étapes: la présentation, le développement, l'approfondissement et la conclusion.

8. Cf. Broadhead et Rist (1976). 
de se mêler au sein des petits ensembles de salariés et de rencontrer directement les dirigeants, car les obstacles bureaucratiques sont moins nombreux dans les TPE. Plutôt que d'aborder les relations d'autorité et d'encadrement dans leur dimension verticale, dirigeants / dirigés, l'immersion et l'observation amènent à s'intéresser aux relations internes au groupe, aux différenciations subtiles qui l'organisent. Elle met en évidence les éléments contextuels et notamment culturels qui pèsent et agissent sur les comportements et les pratiques.

\subsubsection{L'objet et les acteurs étudiés}

Les TPE innovantes à haute technicité ainsi que les TPE du secteur informel ne sont pas prises en compte dans notre étude. Devant la diversité de TPE, de modalités de création, de modes de fonctionnement, il convient de s'appuyer sur un fil conducteur. Il s'agit donc de privilégier la logique d'action du dirigeant d'une organisation située dans les secteurs de l'artisanat et de la petite transformation, des services à la personne ou aux institutions (commerces, garages, restauration...). Nous estimons que ces TPE sont les plus représentatives des réalités et dynamiques industrielles de la région concernée.

Concernant les dirigeants interrogés, nous avons répertorié leurs caractéristiques personnelles et professionnelles. Les éléments regroupés permettent de connaître leurs origines, leurs motivations, leur formation ainsi que leur parcours professionnel.

Deux groupes de dirigeants se distinguent. Le premier est composé de dix dirigeants. Ces derniers s'insèrent dans la «logique patrimoniale». Les dirigeants sont des PIC ( $c f$. supra). L'âge est compris entre 50 et 60 ans. Généralement, ils sont dirigeants d'une entreprise familiale. Leur atout réside dans l'aide fournie par leurs parents. Ils sont issus des zones montagnardes de la région et très souvent du Rif. Ils estiment que le réseau relationnel est très important dans leur vie professionnelle; d'ailleurs, ils le considèrent comme un filet de sécurité. Ces dirigeants ont souvent abandonné leurs études sous la pression parentale afin d'intégrer l'entreprise familiale.

Le schéma est souvent le même. Le père crée l'entreprise et cède l'entreprise à son fils qu'il désigne pour gérer et développer l'activité. Ce sont donc des «héritiers». Nos dirigeants ont exercé différentes fonctions. Ils ont accumulé beaucoup d'expériences tout au long de l'activité de l'entreprise qui a très peu évolué au cours du temps. Nos dirigeants sont essentiellement animés par le maintien de leur activité sur un marché donné tout en fonctionnant par coups et opportunités. 
Le deuxième groupe rassemble cinq de nos dirigeants. Ils sont âgés de 35 à 45 ans. Ils s'insèrent également dans une logique patrimoniale. Toutefois, les logiques entrepreneuriales et managériales sont présentes. Ils sont PIC avec une bonne dose de CAP ( $c f$. supra). Ils s'efforcent de diversifier leurs activités en proposant des produits et des services innovants. Ils ont intégré les technologies de l'information et de la communication lesquelles ont bouleversé leurs activités. Ils ont tissé des liens avec des entrepreneurs espagnols, hollandais, français et développent une vision transnationale. Leurs téléphones ne cessent de passer du réseau marocain au réseau espagnol. Ils sont issus de familles de Fassi et des Casaoui $^{9}$ arrivées discrètement dans les années 1970. Ces cinq dirigeants ont bénéficié de privilèges qui sont fondés sur des recommandations et de la cooptation. L'un d'eux a bénéficié de la position favorable de son père bien introduit dans les sphères politiques. Nos cinq dirigeants sont engagés dans le même réseau associatif. Ils considèrent que la région du Nord et surtout Tanger ${ }^{10}$ vibrent comme une ruche. Ils veulent gagner de l'argent sans s'user au travail. Pour eux, le capitalisme est tout sauf un mouvement tranquille; bien au contraire, il bouleverse et excite.

Les salariés interrogés sont des techniciens, des ouvriers qualifiés, des agents administratifs et des agents d'exécution. Ils constituent généralement la composante la plus importante des effectifs des TPE au Maroc. La part des cadres est très faible (deux personnes).

\subsubsection{Le décryptage et la classification des données: analyse thématique}

Dans la présente recherche, l'exploitation des données qualitatives se réalise à travers une analyse thématique de la restitution des informations recueillies pendant les entretiens; données ouvertement, elles sont volontaires. Durant la première lecture, les principales phrases exprimées par les acteurs sont recensées. Ces dernières sont nommées phrases témoins (10 à 30 par entretien); suit une classification de ces dernières dans des thèmes et sous-thèmes par population interrogée. Pour le dirigeant, il s'agit du style d'autorité et d'encadrement des dirigeants, de sa perception du rôle du dirigeant (ce que doit ou devrait être un dirigeant de TPE), de ses pratiques d'animation des relations humaines, des buts recherchés et des logiques d'action. Concernant le salarié, les thèmes s'articulent autour du comportement et des pratiques du dirigeant perçus par les salariés, de leurs

9. Les Fassi sont les habitants de la ville de Fez et les Casaoui, ceux de Casablanca. 10. Cf. Peraldi, 2008. 
attentes dans ce domaine et de leurs critères de légitimité (ce que doit ou devrait être un dirigeant de TPE; sur quels éléments repose la légitimité du dirigeant? quels liens avec l'éthos?...).

\section{Discussion, implication managériale et limite: les «analyses»"}

Sur les bords de la Méditerranée, le fonctionnement des entreprises, le choix des dirigeants et le contrôle de l'action s'inscrivent généralement dans un mode de gouvernance administré et en réseau. Le modèle administré est essentiellement pris en charge par la puissance publique tandis que le modèle en réseau s'organise à partir des liens personnels et sociaux articulés à partir de la famille, le clan, l'appartenance religieuse... (Orsoni et Pérez, 2006). La dimension financière n'est pas la seule valeur dominante. Le dirigeant de TPE veille avant tout, de manière directe ou indirecte, à maintenir une cohésion sociale et sociétale. Il adopte des logiques d'action et un mix de relations humaines qui semblent les mieux acceptées socialement. Il s'agit d'une responsabilité de proximité et incrustée.

\subsection{Le style d'autorité/encadrement et les pratiques d'animation des relations humaines du dirigeant}

Le dirigeant des TPE est souvent considéré comme une personne autoritaire/ tyrannique ou paternaliste, subsistance d'une forme d'organisation considérée comme «archaïque», du simple fait que la confiance, les relations personnelles et émotionnelles seraient adoptées au détriment de la compétence formelle et formalisée, de la professionnalisation, de la planification... Sans nier les formes d'asservissement réelles qui peuvent exister dans toutes les entreprises, ce style d'autorité et d'encadrement offre la possibilité d'intégrer des tâches à des affects. Les relations reposent sur la confiance réciproque dans la mesure où les règles implicites prédominent sur les règles explicites. Cela exige alors de la fluidité relationnelle regroupant la présence, la proximité, l'échange, une capacité de déduction des règles à partir de signaux faibles et subtils et du soutien réciproque.

11. Compte tenu de la taille de l'échantillon, les analyses présentées ne peuvent être étendues et extrapolées à l'ensemble de la population des TPE. Les interprétations ne dépassent pas le caractère exploratoire de l'étude et nos recherches ciblent uniquement le Maghreb. Nos résultats dépendent des particularités du contexte étudié. Malgré nos fondements théoriques, nos conclusions souffrent d'un manque de comparaisons avec d'autres régions de la Méditerranée. 


\subsubsection{Ce que doit être un dirigeant: regards croisés}

La majorité des dirigeants affirment: "Nous sommes au Maghreb et le dirigeant est un chef, ça se respecte. L'autorité n'est pas liée à la compétence ni au pouvoir financier.» Dans les TPE observées, l'exercice de l'autorité est lié à l'ancienneté ou à l'appartenance à une famille. «Il est difficilement admis que les très jeunes soient dans les sphères de décision, quel que soit leur niveau de compétences. Ne pas tenir compte de ce paramètre, c'est s'exposer à de nombreux blocages. » Tous les dirigeants rencontrés estiment qu'un dirigeant désigne la capacité d'une personne à «fédérer les autres autour de sa vision». Dans la région nord du Maroc, les différentes cellules sociales (clan, famille, entreprise) fonctionnent souvent sous l'autorité reconnue d'une seule personne. Il appartient au dirigeant de légitimer sa position, en évitant les conflits, pour installer durablement son autorité sur les travailleurs. «En général, le dirigeant appartient à une communauté qui l'aide quand il en a besoin, et qu'il doit à son tour aider dès qu'il le peut.» L'autorité doit reposer sur l'esprit communautaire: «Chez vous, le nombre croissant de Team Building témoigne de l'importance de la progression de la notion d'équipe. Dans notre culture, l'existence d'un esprit communautaire, la solidarité et l'esprit d'échange existent déjà à l'état naturel. Il appartient au dirigeant d'exploiter cette prédisposition pour monter des équipes performantes collectivement.» Pour les salariés, le dirigeant représente tout une communauté qui vit au rythme de ses joies et peines professionnelles. "Un licenciement est souvent un drame social qui entraîne avec lui la déchéance de tout un groupe d'individus aussi bien dans l'entreprise qu'en dehors.»

«Nos dirigeants savent que s'ils nous virent [...] le lendemain matin, nous leur amenons nos enfants. Ils ont conscience du mal qu'ils peuvent occasionner. » Les dirigeants et les salariés rencontrés ne sont pas partisans d'une productivité élevée; ils n'en comprennent pas la finalité. En général, ils se satisfont d'un niveau de productivité permettant de dégager une marge bénéficiaire et d'assurer la vie de leur structure. Ils ne sont pas préoccupés par la compétition avec leurs concurrents, qu'ils perçoivent comme «des gens ayant le droit de vivre et de profiter de leurs activités». Mieux, les communautés sont si interreliées qu'encourager la compétition et l'élévation de la productivité peut être perçu comme une volonté délibérée de mettre «à mort son concurrent et l'équilibre sociétal».

\subsubsection{Ce que font les dirigeants: les logiques d'action}

Pour nombre de dirigeants des TPE étudiées, la taille de la structure et l'effectif sont des éléments fondamentaux dans la pratique de leur gestion. La perte de cette petite taille est perçue comme un frein au développement 
et comme propice à la rupture de la nature des liens relationnels. Globalement, les dirigeants rencontrés sont le plus souvent animés par une dynamique entrepreneuriale, voire managériale impulsée par la mondialisation; néanmoins, cette évolution est tissée sur une toile patrimoniale. Les pratiques de recrutement sont le plus souvent basées sur le bouche à oreille. L'informel domine l'entreprise et les pratiques s'appuient sur la subjectivité. Les «systèmes» d'appréciation sont subtils et informels: "C'est une évaluation collective. Nous félicitons les réussites et on tait les échecs. Ce sont des messages que les collaborateurs comprennent. Ils rectifient très vite et se corrigent seuls. » L'appréciation se manifeste plus par des rétributions qui s'appuient sur des valeurs communes. Ainsi, l'appartenance de l'individu à une communauté empêche la promotion de mesures incitatives à la performance individuelle. "Ici pas de désignation du meilleur employé du mois... Ce genre de mesures entraîne un climat social délétère et des conflits permanents. La suspicion entre les employés augmente et brise la chaîne de solidarité existante.» Un autre répondant précise: «Nous pouvons désigner le meilleur employé à condition qu'il partage avec ses proches les récompenses qui y sont attachées. » La récompense est ressentie comme un opprobre sur la communauté, qu'elle soit intra- ou extra-entreprise. En revanche, une faute professionnelle commise par un agent engagera la responsabilité de toute son équipe de travail.

Les dirigeants interrogés sont également portés par une logique managériale qui privilégie les qualités de gestionnaire. Dans les discours, une formalisation des pratiques existe avec des relations plus procédurales et rationnelles; la norme y est importante. Toutefois, la dimension «intellect» baigne dans un univers $\mathrm{d}^{\prime}$ " affect».

L'homme «méditerranéen» (dirigeant ou salarié) n'est pas un aventurier solitaire. Il évolue au sein de relations de proximité (Marchesnay, Kammoun et Karray, 2006). Il travaille au sein d'un territoire délimité (ville, village; proximité géographique) dans lequel il entretient des relations de sociabilité fortes (sympathie, empathie; proximité sociale). Il adhère aux valeurs sociétales propres à cet espace (proximité sociétale) et il mène également des activités économiques (proximité économique). Ces effets de proximité expliquent certaines pratiques d'animation des relations humaines: "Personne ne connaît le salaire de personne. Chacun se tait. On ne peut pas se comparer, car tout le monde se connaît. On tourne et on évolue autour des mêmes personnes. La région est petite, la caisse de résonance n'est pas la même, l'écho est plus fort. » En Méditerranée, les réseaux informels dominent l'entreprise. Ce sont des réseaux relationnels centrés sur la personne: «Qui es-tu?», «Qui est ton père?», «De quel village, ville es-tu? ». Le positionnement dans le réseau est très important. 


\subsubsection{Comment ils le font: les logiques d'exécution. Une fluidité relationnelle facilitée par la présence, l'échange et le sens de la mesure}

Selon nos observations et nos entretiens, les dirigeants sont avant tout caractérisés par le fait d'être omniprésents. Ils consacrent beaucoup de temps à observer, questionner, à écouter les uns et les autres, ce qui les amène à avoir une bonne connaissance de la réalité du quotidien. Ils s'appuient sur un vaste réseau de communication informelle qui permet d'échanger, de toucher le réel au plus près, de résoudre des problèmes de façon très spontanée et en temps réel. Cela rejoint et dépasse le principe de management baladeur ou du management by walking around développé par Peters et Waterman (1983), qui consiste à quitter fréquemment «le pont du navire et à abandonner la longue vue» afin «de se balader dans le navire avec l'équipage».

Les dirigeants dont nous parlons vont au-delà du management baladeur, car ils éliminent les formalités et les procédures pour se rapprocher et rendre plus directes, plus nombreuses et plus fluides leurs relations avec leurs collaborateurs. Il s'agit en fait d'un décloisonnement hiérarchique. La gestion des hommes est d'abord une histoire de relations, de contacts, d'écoute et de face à face. La mise en relation n'impose pas aux acteurs de partager les mêmes intérêts, mais elle requiert l'aptitude à élaborer des passerelles grâce à la parole. Cette fluidité relationnelle semble en outre faciliter la maitrise de l'environnement interne de l'entreprise grâce à une collecte directe des informations et des suggestions. Elle permet de repérer les non-dits qui en disent souvent long et de mieux cerner les différentes personnalités du groupe. Cette façon de faire peut permettre également de mieux maîtriser le rythme temporel, en étant beaucoup moins dans l'urgence et la précipitation. Ces dirigeants ont le sens de la mesure, de la recherche de ce «juste milieu » aristotélicien si cher aux sociocultures non occidentales, en général, et à la Méditerranée orientale en particulier (Frimousse et Aktouf, 2011). Pour tous les dirigeants interrogés et observés, un dirigeant se doit, à travers son autorité et son encadrement, «comprendre et déchiffrer les inquiétudes, les émotions, les sensations, les espoirs et les attentes des salariés». Il s'agit d'écouter et de comprendre. Cela dit, les qualités humaines des dirigeants doivent être reconnues et appréciées.

\subsection{Les fondements de la légitimité d'action des dirigeants: les effets de l'encastrement}

Nos dirigeants sont, comme tous les dirigeants, en quête de légitimité auprès de leurs salariés et des autres "parties prenantes». Mais il apparaît que dans le contexte ici exposé ( $c f$. supra), leurs actions ne doivent pas répondre 
seulement à des exigences de type «performances économiques»; elles doivent également (sinon plus) répondre à des attentes bien plus spécifiquement socio-humaines, relevant de valeurs et normes sociétales bien plus ancrées dans l'informel, le socio-organique, le socio-affectif. Nous appellerons cela «l'acceptabilité morale» du dirigeant: une sorte de jugement social «favorable» à obtenir des employés et des parties prenantes (fournisseurs, clients), aussi importante que la performance économique ou financière. Comme dans le cas de l'autorité «traditionnelle» wébérienne, notre dirigeant doit promouvoir, renforcer et protéger des valeurs, des façons d'agir et de gérer... qui tiennent compte de traditions et coutumes ancestrales et régionales (et s'y enracinent), autant que tenter de se familiariser avec des méthodes de management «moderne».

\subsubsection{Une hybridation des logiques d'action: entre sphère de production économique et sphère domestique}

La rencontre entre un éthos non figé et les nouvelles exigences posées par la mondialisation semble exercer une influence sur certains dirigeants au point de livrer, dans certains cas, une configuration d'actions hybrides nouvelle. Certains dirigeants semblent choisir ce qui leur convient tout en greffant ces nouveaux éléments à leurs éthos. Ces dirigeants réalisent un ajustement entre la mondialisation inévitable et les aspirations «individuelles» et «communautaires » à un maintien vital de leurs sphères culturelles et contextuelles. L'hybridation invite à emboîter les cultures, les logiques d'action les unes dans les autres afin de tirer profit de ce croisement. Cela correspond en fait à un brassage des différences. Cette pensée de l'entre-deux permet d'éviter les réactions brutales suscitées par l'irruption d'éléments nouveaux.

L'hybridation semble permettre de réaliser un ajustement entre l'inévitable globalisation et les réalités locales. On a alors affaire à une imbrication entre la sphère de production économique et la sphère plus «domestique » (toujours au sens wébérien) mais élargie au microcosme civil de la TPE qui forme alors une «communauté» de type «domestique» quasi familial. L'une des phrases les plus significatives prononcées par les dirigeants dont nous parlons est assez éloquente en ce sens: «Nous sommes tous dans le même plateau. Les verres sont mes salariés et mon entreprise, la théière. Je dois veiller à ce que la théière ne soit pas vide et que les verres ne soient ni vides ni pleins. » Il s'agit là d'une métaphore qui exprime bien au moins deux dimensions très intéressantes pour notre propos: 1) nous avons affaire à un dirigeant qui affirme son humilité, il n'est pas l'être tout-puissant portant l'exclusivité du «savoir gérer»; et 2) il affirme avoir à remplir un devoir de nature pater familias: les «théières» (employés) ne sont pas traités comme ayant 
uniquement la charge de remplir les verres (produire), ils doivent être convenablement traités, considérés... de sorte que la situation globale de travail (plateau théière et verres) soit constamment à son «optimum». On peut voir ici, en filigrane, les rôles de pourvoyeur de ressources, de figure de proue, de défenseur et porte-parole... des employés établis par Mintzberg (1973).

\subsubsection{Un dirigeant mortel parmi les mortels}

Nos dirigeants observés et interrogés acceptent et se font un devoir de se rapprocher de leurs collaborateurs. Ils en méritent ainsi l'attachement et l'estime, bases de légitimation du rôle de dirigeant. Toute relation basée uniquement sur le contrôle et le respect des clauses du «contrat rationnel» classique est vouée à l'échec. Le véritable contrat qui est ici en jeu est plutôt d'ordre personnel, informel et tacite - psychologique. Il englobe rapports sociaux marchands, relations de type «pater familias», exercice de pouvoir légitimé, soutien et réciprocité. Ce qu'exprime fort bien cette phrase d'employé: "Il [dirigeant] est avec nous toute la journée, il travaille, il nous parle. Au niveau des heures, il nous arrange en cas de besoins... Pour les mariages, les fêtes, les décès..., il apporte un soutien financier... C'est une main bienveillante...» C'est là une situation qui favorise l'éclosion d'un dirigeant s'adressant à des personnes et non à des «ressources».

Le dirigeant ici se comporte en simple mortel parmi les mortels! Cette dé-déification et re-personnalisation s'insèrent dans un fonds culturel proche de la «civilisation du chacun à sa place » (au sens où chacun a son importance) de la société japonaise, déjà décrite par Benedict (1995). La "réserve-honte » et la pudeur-modestie, deux attitudes-émotions proches, constituent une norme sociale très forte en Méditerranée du Sud. La réserve-honte surgit lorsque certaines valeurs liées au respect de l'autre, à son amour propre, à son honneur, à une sorte de hantise de ne jamais «faire perdre la face» à quiconque, sont bafouées ou offensées. En cas de manquements à ce genre de règles implicites, nos dirigeants et leurs salariés connaissent ce qu'Aristote a nommé aiskhunè, soit une émotion de honte-atteinte à l'honneur-amour propre... liée à toute action considérée comme condamnable (Boquet, 2009). Ce regard individuel collectif «contraignant» est aussi, et par là même, une reconnaissance existentielle accordant à chacun le statut de «sujet».

\subsubsection{La honte et la réciprocité}

Dépassant «les ressorts» du sentiment de culpabilité, dirigeants et salariés semblent évoluer dans un univers de sens régi par un puissant dispositif émotionnel et moral: la honte (hachma, hchouma, vergogna...). La crainte 
du blâme et la honte d'y être exposé sont des remparts contre l'affaiblissement du lien social. Dirigeant et salarié doivent se positionner de façon adéquate dans l'espace social qui constitue une communauté «intime». Les membres de cette communauté s'informent continuellement des affaires des autres. Les référents édictés explicitement ou implicitement par la société supposent l'image de soi et la conscience de soi (Piolat, Hurtig et Pichevin, 1992). Dans nos cas, cette attitude-émotion, réserve-honte... respect de l'amour propre de l'autre..., est un regard critique sur l'environnement social immédiat, intériorisé par des «sujets » animés par une logique de réciprocité. En effet, un salarié qui se sent soutenu et en confiance répondra généralement par des comportements ou des attitudes positives comme l'implication. Cela se retrouve, d'après nos observations, dans l'éthos méditerranéen, et engendre au sein d'entreprises de petite taille, un comportement-besoin de «protection implicite» réciproque. Il s'établit donc une forme de contrat ou de serment implicite qui lie les êtres, les personnes. L'homo «reciprocus» a un air de famille avec l'homo "oeconomicus», mais il gagne à condition de ne pas être intéressé à gagner, de ne pas adopter le schéme de l'intérêt individuel, de ne pas calculer. Selon nos observations et nos entretiens, ces principes permettent au salarié de recevoir davantage que ce qui leur est contractuellement et statutairement dû: une configuration d'indulgence (la seconde chance); une certaine souplesse dans l'application des règles; l'autorisation d'utiliser pour son usage personnel les outils de l'entreprise.

\section{Conclusion}

Nos travaux se sont orientés vers la Méditerranée et, plus précisément, le Maghreb pour explorer et découvrir le style d'autorité et les pratiques d'animation des relations humaines des dirigeants de TPE. Ces derniers y ressentent fortement l'importance des parties prenantes, car ils sont économiquement et socialement encastrés dans un éthos qui conditionne leur légitimité et leur compétitivité. Les actions des dirigeants du Sud de la Méditerranée œuvrant dans des cadres organisationnels de «petites dimensions» ne doivent pas répondre seulement à des exigences de type "performances économiques», ils doivent également répondre à des attentes plus socio-humaines, relevant de valeurs et normes sociétales bien plus ancrées dans l'informel, le socioorganique, le socio-affectif. Ces constats nous amènent à préciser dans nos futures recherches la relation dirigeant-dirigés au sein des TPE. Au-delà des éléments contractuels organisationnels, en vertu de quelles dispositions les salariés, mais aussi les dirigeants se sentent-ils «tenus» d'entrer dans une interaction supérieur-subordonné? Pourquoi et comment se lie-t-on à un dirigeant et s'implique-t-on pour lui? Les notions d'honneur et de 
légitimité semblent constituer des pistes de réponses à explorer dans nos futurs travaux afin de mieux comprendre les liens entre la petite entreprise et l'éthos méditerranéen.

\section{Bibliographie}

ADDI, L. (2004), L'anthropologie du Maghreb selon Berque, Bourdieu, Geertz et Gellner, Paris, Awal Ibis Press.

ADLER, P. et E. ROCHFORD (1986), «The politics of participation in field research», Journal of Contemporary Ethnography, vol. 14, n 4, p. 363-376.

AKTOUF, O. (1986), «Une vision interne des rapports de travail: le cas de deux brasseries», Le travail humain, vol. 49, n 3 , p. 237-248.

ALLALI, B. (2010), «Entrepreneuriat maghrébin au Québec: un entrepreneuriat par nécessité ? », $\mathrm{X}^{\mathrm{e}}$ Congrès CIFEPME, Bordeaux IV.

BACHELOT, C. (2011), «L'ethnographie des dirigeants de partis. Le cas du parti socialiste», Genèses, n 83 , p. 118-132.

Benedict, R. (1995), Le chrysanthème et le sabre, Arles, Picquier Poche.

BERQUe, J. (1953), Qu'est-ce qu'une tribu nord-africaine?, Paris, Colin.

BERQUE, J. (1955), Structures sociales du Haut-Atlas, Paris, Presses universitaires de France.

BERRY, M. (1987), Vers une technologie invisible?, Paris, Cahier du CRG.

BLOK, A., C. BROMBERGER et D. AlBerA (2001), L'anthropologie de la Méditerranée, Paris, Maison méditerranéenne des sciences de l'homme.

BOQUET, D. (2009), «Introduction», Rives méditerranéennes, n 31, p. 7-16.

BOURDieU, P. (2001), Sociologie de l'Algérie, 8e édition, Paris, Presses universitaires de France.

BRINCKMANN, J., D. GRICHNIK et D. KAPSA (2010), «Should entrepreneurs plan or just storm the castle? A meta-analysis on contextual factors impacting the business planning - performance relationship in small firms », Journal of Business Venturing, vol. 25, n 1 , p. 24-40.

BROADHEAD, R.S. et R.C. RIST (1976), «Gatekeepers and the social control of social research», Social Problems, vol. 23, n 3, p. 325-336.

CHANLAT, J.F. (2005), «La recherche en gestion et les méthodes ethnosociologiques», dans P. Roussel et F. Wacheux (dir.), Méthodes de recherche en sciences humaines et sociales, Bruxelles, De Boeck.

CROZIER, M. (1963), Le phénomène bureaucratique; essai sur les tendances bureaucratiques des systèmes d'organisation modernes et sur leurs relations en France avec le système social et culturel, Paris, Éditions du Seuil. 
DEJOUX, C. (2001), «Organisation qualifiante et maturité en gestion des compétences », Personnel, n 369 , p. 61-67.

ÉTIENNE, B. (1999), Une grenade entrouverte, Avignon, Éditions de l'Aube.

FAVRET-SAADA, J. (1966) «La segmentarité au Maghreb», L’Homme, vol. 6, nº 2. p. 105-111.

Fourcade, C., V. Gallego, M. Polge et L. SAOudi (2010), «De la GRH à l'animation des relations humaines. Le cas des entreprises artisanales », Revue Management et Avenir, p. 141-157.

FOURCADE, C. et M. POLGE (2008), «Sur les parcours de la démarche innovante: pour une approche prospective et personnalisée», Annales 2008-2009 du Réseau Artisanat Université, ISM, Paris.

FRIMOUSSE, S. (2011), «Méditerranée: rupture ou unité ?», Actes de l'université de printemps de l'IAS, Agadir.

FRIMOUSSE, S. et O. AKTOUF (2011), «La Méditerranée: un “orient” plus prometteur pour le leadership?», dans Tous leaders, Eyrolles, Éditions d'Organisations.

FRIMOUSSE S. et J.M. PERETTI (2006), «L'émergence d'une gestion des ressources humaines hybride au Maghreb», Revue française de gestion, vol. 32, aoûtseptembre, p.149-159.

GArfinKel, H. (1957), Studies in Ethnomethodology, New York, Prentice Hall.

GEERTZ, C. (1973), The Interpretation of Cultures, New York, Basic Books.

GÉLARD, M.L. (2003), Le pilier de la tente, Paris, Éditions de la Maison des sciences de l'homme.

GELLNER, E. (2003), Les saints de l'Atlas, traduit de l'anglais par Paul Coatelen, Saint-Denis, Éditions Gianni Albergoni.

GLASER, B. et A. STRAUSS (1967), The Discovery of Grounded Theory: Strategies for Qualitative Research, Chicago, Aldine de Gruyter.

GOULDNER, A. (1960), «The norm of reciprocity: a preliminary statement», American Sociological Review, vol. 25, nº 2, p. 161-178.

GuillemeTtE, F. (2006), «L'approche de la Grounded Theory: pour innover?», Recherches qualitatives, vol. 26, $\mathrm{n}^{\circ} 1$, p. 32-50.

JULIEN, P.-A. (1997), Les PME: bilan et perspectives, Paris, Economica.

JULIEN, P.-A., M. MARCHESNAY et H.V. MADADO (2010), «Pour une théorie entrepreneuriale tenant compte des différences culturelles », $\mathrm{X}^{\mathrm{e}}$ Congrès CIFEPME, Bordeaux IV.

KORSGAARD, S. et A.R. ANDERSON (2011) «Enacting entrepreneurship as social value creation », International Small Business Journal, avril, p. 135-151.

LACOSTE, Y. (1998), Ibn Khaldun: naissance de l'histoire, passé du tiers monde, Paris, La Découverte.

LENCLUD, G. (2007), «Naissance de l'histoire, passé du tiers monde, La Découverte...», Terrain, no 9, p. 110-123. 
LiGHT, L. et S. GOLD (2000), Ethnic Economics, New York, Academic Press. MAHÉ DE Boislandelle, H. (1998), GRH dans les PME, Paris, Economica.

MARChESNAY, M. (2002), Pour une approche entrepreneuriale de la dynamique ressources-compétences: essai de praxéologie, Les Éditions de l'ADREG, France, <www-editions adreg.net $>$.

MARCHESNAY, M. (2003), «La petite entreprise: sortir de l'ignorance», Revue française de gestion, Paris, nº 144, p. 107 à 118.

MARCHESNAY, M. (2004), Management stratégique, Les Éditions de l'ADREG, France, $<$ www-editions adreg.net $>$.

MARCHESNAY, M. (2008), «L'hypofirme, vivier et creuset de l'innovation hypermoderne », Innovations, n 27, p 147-161.

MARCHESNAY, M., S.C. KAMMOUN et H.E. KARRAY (2006) «Y a-t-il un entrepreneuriat méditerranéen? », Revue française de gestion, vol. 32, nº 166.

MERTON, R.K. (1965), Éléments de théorie et de méthode sociologique, Paris, Plon.

MinTZBERG, H. (1973), The Nature of Managerial Work, New York, Harper and Row.

ORSONI, J. et R. PÉREZ (2006), «Le management méditerranéen : forme dépassée ou formule d'avenir?», Revue française de gestion, $\mathrm{n}^{\circ}$ 166, p. 95-100.

PAGÈS, M. (2002), La vie affective des groupes - esquisse d'une théorie de la relation humaine, Paris, Dunod.

PARADAS, A. (2007), «Le dirigeant comme levier de la RSE en TPE. Approche exploratoire basée sur l'utilisation de récits et d'une cartographie cognitive», Revue internationale PME, vol. 20, nos 3-4, p. 43-67.

PERALDi, M. (2008), «Tanger transnationale», La pensée de midi, n 23. p. 8-17.

PETERS, T. et R. WATERMAN (1983), Le prix de l'excellence, Paris, InterÉditions.

PIOLAT, M., M.C. HuRTIG et M.F. PICHEVIN (1992), Le soi. Recherches dans le champ de la cognition, Lausanne, Delachaux et Nestlé.

PITT-RIVERS, J. (1997), Anthropologie de l'honneur: la mésaventure de Sichem, Paris, Hachette.

Serarols, C., Z. AldeMiR et H. JOO (2011), «Book review: entrepreneurship and culture», International Small Business Journal, juin, vol. 29, p. 296-298.

SPRADlEY, J.P. (1980), Participant Observation, New York, Rinehart and Winston.

THORNTON, P., D. RIBEIRO-SORIANO et D. URBANO (2011), «Socio-cultural factors and entrepreneurial activity: an overview», International Small Business Journal, avril, p. 105-118.

TORRÈS, O. (2008), «Comprendre le management des PME: le rôle clé de la proximité», Conférence à l'EM, Lyon.

TORRÈS, O. et G. GUEGUEN (2008), «Incidence de la loi proxémique sur la perception de l'incertitude des PME», Revue internationale PME, vol. 21, n 1, p. 93-117. 
VERNAZOBRES, P. (2009), «La mise en place d'un design de la recherche basé sur une approche ethnographique pour investiguer les pratiques confidentielles du coaching », Revue de gestion des ressources humaines, $\mathrm{n}^{\circ}$ 74, p. 42-62.

WEBER, M. (1956), Économie et société, Tübingen, Mohr. 